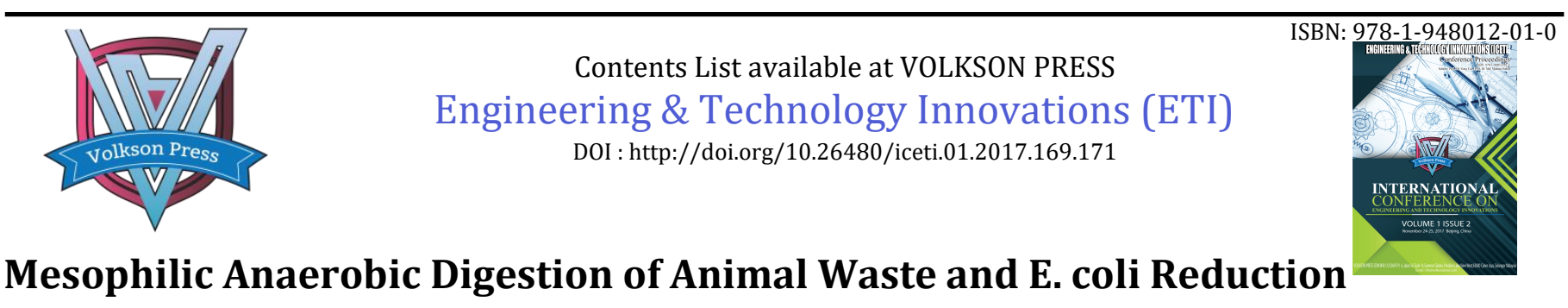

Yi Wang , Pramod Pandey

Department of Population Health and Reproduction, School of Veterinary Medicine, University of California-Davis, USA yyiwang@ucdavis.edu (Yi Wang); pkpandey@ucdavis.edu (Pramod Pandey)

This is an open access article distributed under the Creative Commons Attribution License, which permits unrestricted use, distribution, and reproduction in any medium, provided the original work is properly cited

\section{ARTICLE DETAILS}

\section{Article History:}

Received 02 october 2017 Accepted 06 october 2017 Available online 11 october 2017

\section{Keywords:}

pathogen, anaerobic digestion, mesophilic temperature, animal waste,

\title{
ABSTRACT
}

Anaerobic digestion (AD) is one of the preferred method for treating animal wastes, and emphasize of this study is to investigate $\mathrm{E}$. coli reductions in AD system. The AD is widely applied in developing countries such as China and India. The AD has been also used in developed countries such as USA and Germany for treating animal waste, particularly, dairy manure. Both thermophilic $\left(\approx 50-55^{\circ} \mathrm{C}\right)$ temperature and mesophilic temperature $\left(\approx 35-37{ }^{\circ} \mathrm{C}\right)$ AD systems are used; however, mesophilic $\mathrm{AD}$ is more common than the thermophilic AD because of no requirement for external energy to increase the temperature in mesophilic AD system. The advantages of AD system include the productions of biogas (a source of renewable energy), and digestate (used as a fertilizer). Another benefit of the AD is that it helps in controlling animal waste born pathogen and pathogen indicator (such as E. coli) reductions. Additional studies are needed to investigate E. coli reductions in the AD system. This study was focused on understanding of E. coli reduction in the $\mathrm{AD}$, which was operated in mesophilic temperature conditions. Subsequently, models were developed to calculate E. coli reductions. Two different types of animal manure feedstocks were used for calculating E.coli reduction. In the first feedstock, liquid slurry from solid manure (scrapped manure) was prepared in the lab, while the second feedstock includes flushed dairy manure (i.e., liquid manure) collected from dairy lagoons located in dairy farms. Results of observation and model showed the AD system reduces E. coli level substantially; however, the reduction level depends on the incubation time.

\section{Introduction}

Increasing waste from animal-agriculture system is a serious issue, which poses numerous challenges in terms of environmental contamination such as microbial contamination in ambient water $[1,2]$. Controlling microbial contamination requires identifying and implementing the appropriate animal manure treatment methods capable of reducing animal waste borne microbial pathogen levels. Both aerobic and anaerobic digestions are used for treating animal waste $[1,2,3]$. The anaerobic digestion (AD) of animal manure, however, is a preferred method because it has significant importance in terms of biogas production - a source of energy [1]. The application of AD in both developing and developed countries for biogas production and manure treatment is well established and has been implemented all over the world. Further, there is renewed interest in the uses of AD system because of its role in reduction of greenhouse gas (GHG) emissions. As an example, the installation of dairy digesters for treating animal manure for GHG reduction in relatively larger dairy farms in the California Central Valley has received considerable interest among dairy farmers, and to facilitate such initiative, financial support is provided by the government [3]. Similar initiatives to increase the installation of AD systems for treating livestock manure in rural areas of India and China are in place for decades. For example, Ministry of New and Renewable Energy (MNRE) in India supported the setup of more than 4 million family type biogas plant (i.e., anaerobic digesters) in India [4]. Similarly, more than 30 million household biogas plants are installed in China [5]. Biogas Institute of Ministry of Agriculture (BIOMA) helped in developing biogas research and application program in China for treating waste of the livestock and poultry industries [6]. A large number of applied research has been conducted to understand the anaerobic digestion process and enhance biogas production during $\mathrm{AD}$ process $[1,5,6,7,8,9,10]$. Another important benefit of the AD system is that it reduces animal waste-borne pathogens. In previous studies, however, understanding of the role of AD on pathogen reductions has received relatively less emphasis. The objective of this study was to investigate pathogen/pathogen indicator ( $E$. coli) reductions during the anaerobic digestions of dairy manure.

\section{Methods}

A series of bench-scale anaerobic digestion experiments were conducted to understand $E$. coli reductions during anaerobic process. The experiments were executed at mesophilic temperatures $\left(\approx 35-37{ }^{0} \mathrm{C}\right)$. Two sets of $\mathrm{AD}$ experiments were undertaken using two different types feedstocks collected from two different geographic locations. In the first set of experiment (Feedstock 1), fresh manure was collected from Iowa State University's dairy facility (100 - 200 dairy cows). The dairy manure was collected 24 hours prior to the start of the experiment. Solid dairy manure was used to prepare the liquid feedstock for the bench-scale anaerobic reactors. Solid manure of $0.498 \mathrm{~kg}$ was mixed thoroughly in $1,500 \mathrm{ml}$ of distilled water to prepare liquid slurry. Fibers and large solid particles of liquid slurry were removed through filtration. Filtered liquid slurry $(\approx 150 \mathrm{~mL})$ was transferred into each anaerobic reactor. Subsequently, the reactor was sealed with a rubber septum. In the second experiment (Feedstock 2), three serum bottles were filled with of flushed liquid manure $(\approx 100 \mathrm{~mL}$ ), and anaerobic environment was created by putting septum on serum bottles similar to previous experiments [1]. For the second experiment, flushed liquid manure was collected from dairy farms located in California Central Valley, USA. The dairy farm in this location houses between 2000 and 3000 dairy cows. In both the experiment, slurry samples from experimental reactors ware collected by inserting syringe through septum at regular intervals $[1,2]$. In Feedstock 1 experiment, $E$. coli enumeration was performed by membrane filtration techniques using standard modified mTEC agar (growth media) [1]. Membrane filters after the filtrations of samples were placed in petri dish with modifified mTEC growth media agar, and it was incubated at $44.5 \pm$ $0.2^{\circ} \mathrm{C}$ for 24 hours. The red or magenta $E$. coli colonies grown in petri dish after incubation were enumerated. In Feedstock 2 experiment, MacConckey II agar with sorbitol (BBL, Becton, Dickinson and Company, Sparks, MD, USA) was used for E. coli enumeration. MacConckey II agar, a selective and differential media for the detection of sorbitolnonfermenting $E$. coli is widely used for the enumeration of $E$. coli colonies. After plating with sample slurry [using Bacteriological Analytical Manual (BAM) procedure], MacConckey agar plates were incubated at $37^{\circ} \mathrm{C}$ for 24 hours [2]. 


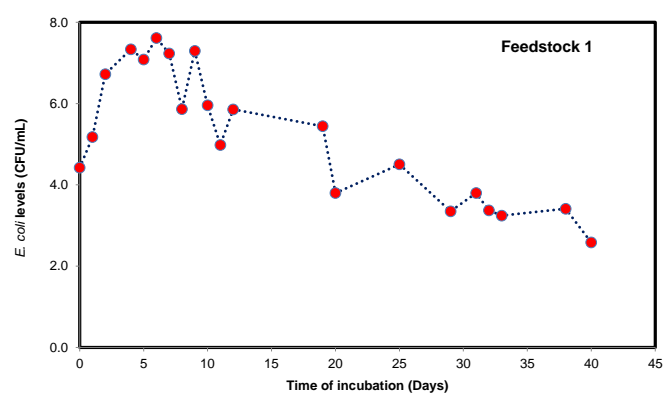

Figure 1a: E. coli reduction and incubation time for Feedstock 1

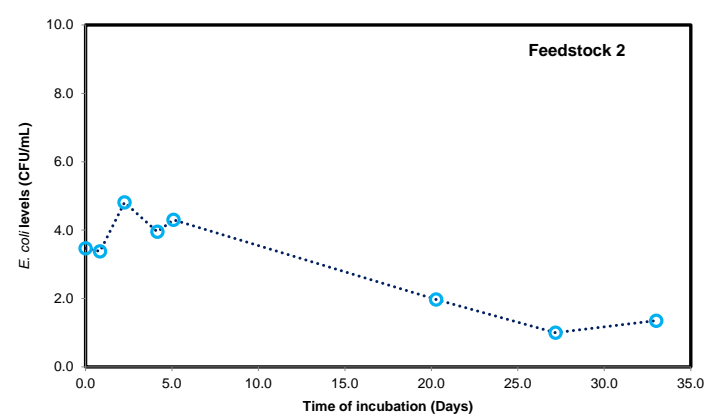

Figure 1b: E. coli reduction and incubation time for Feedstock 2

The growth of pink colonies was enumerated as E. coli using colony counters $[1,2]$. Details of the $E$. coli enumeration, sample preparation, and dilution are provided elsewhere $[1,2]$. Subsequently, based on the observation data, linear models were developed for each feedstock to calculate time required for $E$. coli reductions (i.e., time and $E$. coli reduction relationships). Calculations from Model 1 (based on Feedstock 1) and Model 2 (based on Feedstock 2) were combined to calculate the average values for estimating the time required for E.coli reductions at various incubation period.

\section{Results and Discussion}

The change in $E$. coli levels in Feedstock 1 is shown in Figure 1a. Initial $E$. coli level was in 4.4 orders of magnitude, and final E. coli level became 2.6 orders of magnitude in 40 days of anaerobic digestion. The change in $E$. coli levels in Feedstock 2 is shown in Figure 1b. The initial E. coli level in Feedstock 2 was 3.5 orders of magnitude, and the final E. coli level was 1.4 orders of magnitude after 33 days of anaerobic digestion. In both experiments, an increase in E. coli level was observed between Day 2 and Day 5. This growth in initial phase of experiments can be considered as an effect of acclimatization and perturbation, caused by anaerobic environment. After the initial growth, E. coli reduction was consistent in both experiments/feedstock.

To investigate the relationships between incubation time and E. coli reductions, we developed linear models, which include the data of $E$. coli levels beyond Day 2 (excluding the acclimatization period between 0 and Day 2). Figure 2a shows the linear relationship between incubation days and E. coli levels for Feedstock 1, and Figure 2b shows relationship between incubation days and E. coli levels for Feedstock 2. Model 1 based on Feedstock 1 resulted $R^{2}$ value of 0.87 , and Model 2 based on Feedstock 2 resulted $R^{2}$ value of 0.93

While using the Model 1 and Model 2 for calculating E. coli reductions, we simulated the E. coli reductions with respect to time. Simulation was conducted for reductions of E. coli from 8-orders of magnitude to 0.5orders of magnitude. Model results indicate that initial concentrations of E. coli of 8-orders of magnitude can reach to 6-orders (2-order reductions) of magnitude in 16-17 days of anaerobic digestion. The E. coli levels can be reduced by 4-orders of magnitude in 32-33 days of anaerobic digestions.
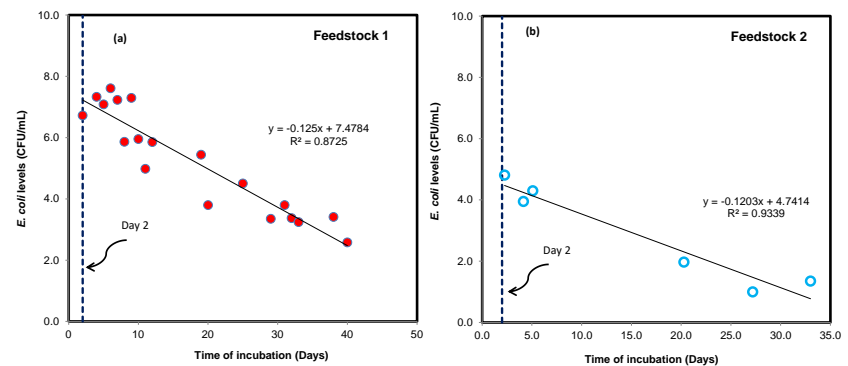

Figure 2: a) model for E. coli reduction in Feedstock 1; b) model for $E$. coli reduction in Feedstock 2.

While using the Model 1 and Model 2 for calculating E. coli reductions, we simulated the E. coli reductions with respect to time. Simulation was conducted for reductions of $E$. coli from 8-orders of magnitude to 0.5 orders of magnitude. Model results indicate that initial concentrations of E. coli of 8-orders of magnitude can reach to 6-orders (2-order reductions) of magnitude in 16-17 days of anaerobic digestion. The E. coli levels can be reduced by 4-orders of magnitude in 32-33 days of anaerobic digestions.

Table 1: Descriptions of E. coli reductions in Feedstock 1 and Feedstock 2

\begin{tabular}{|c|c|c|c|c|c|}
\hline \multirow[b]{3}{*}{$\begin{array}{l}\text { Initial E. coli } \\
\text { levels }\end{array}$} & \multicolumn{2}{|c|}{$\begin{array}{l}\text { Observations [Log } \\
\text { (CFU/mL)] }\end{array}$} & \multicolumn{3}{|c|}{ Predictions [ $\log (\mathrm{CFU} / \mathrm{mL})]$} \\
\hline & Feedstock 1 & Feedstock 2 & $\begin{array}{r}\text { Model } \\
1\end{array}$ & $\begin{array}{r}\text { Model } \\
2\end{array}$ & $\begin{array}{r}\text { Averag } \\
e\end{array}$ \\
\hline & 4.4 & 3.5 & 8 & 8 & 8 \\
\hline Final E. coli levels & 2.6 & 1.4 & 0.5 & 0.8 & 1 \\
\hline \multicolumn{6}{|c|}{ Calculated days for E. coli reductions } \\
\hline & & & Days & Days & Days \\
\hline 2-order reduction & - & - & 16 & 17 & 16 \\
\hline 4-order reduction & - & - & 32 & 33 & 33 \\
\hline 6-order reduction & - & - & 48 & 50 & 49 \\
\hline 7 -order reduction & - & - & 56 & 58 & 57 \\
\hline
\end{tabular}

Table 1 shows the descriptions of E. coli reduction for Feedstock 1 and Feedstock 2. Corresponding results for Model 1, and Model 2 are summarized in the table. Results showed that $E$. coli levels can be reduced from 8 orders of

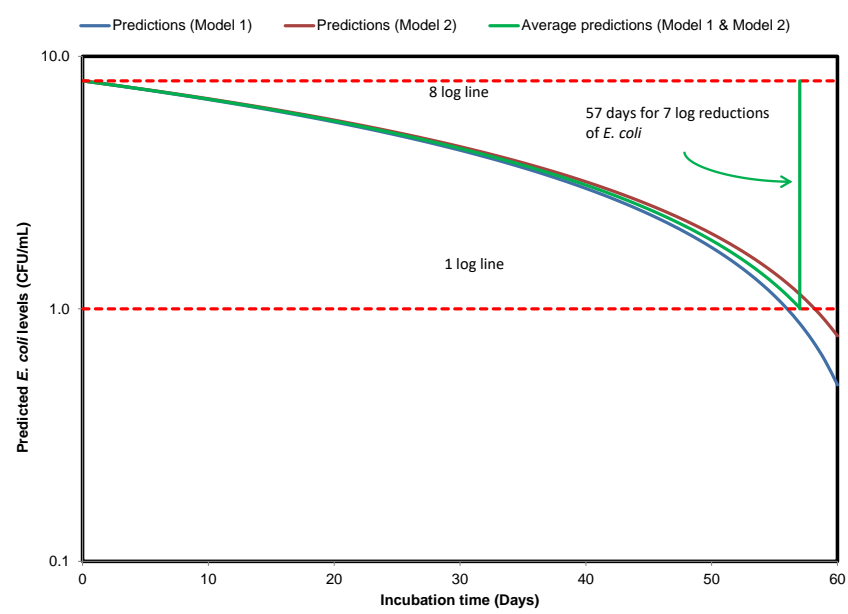

Figure 3: Simulations of E. coli reductions in mesophilic anaerobic digestion of liquid dairy manure.

magnitude to 2-orders of magnitude in 48-49 days of anaerobic digestions. $E$. coli reductions from 8-order of magnitude to 1 -order of magnitude can be achieved in 56-58 days. Figure 3 shows the calculations for E. coli reductions.

Results (Figure 3) showed that approximately 57 days is needed for E. coli reductions from 8-orders of magnitude to 1 -orders of magnitude. These results of Model 1 and Model 2, and the average of results from Model 1 and Model 2 is shown in Figure 3. The results of Model 1 and Model 2 were comparable. The graph shown in Figure 3 can be potentially useful for estimating the $E$. coli reductions in mesophilic anaerobic digestions at various stages of incaution time of anaerobic digestion. 


\section{Conclusions}

This research was focused on understanding of the impacts of anaerobic digestion on E. coli reductions. E. coli reduction in anaerobic digestion of liquid dairy manure was investigated in two feedstocks at mesophilic temperature. Results from bench-scale experiments were used to develop linear models for calculating $E$. coli reductions. Results showed that anaerobic digestion has considerable impacts on E. coli reductions. During acclimatization period (2- 5 days of incubation), E. coli levels were increased, and beyond acclimatization phase, the reductions were relatively consistent. Modeling results showed that E. coli levels can be reduced from 8-orders of magnitude to 4-orders of magnitude in 33-34 days of anaerobic digestion. To reduce $E$. coli levels from 8-orders of magnitude to 1 -order of magnitude, 56-58 days of anaerobic digestion may be needed. These results suggest that anaerobic digestion has a potential use for reducing animal waste borne E. coli. The level of reductions in anaerobic digestion of dairy manure depends on the incubation period.

\section{REFERENCES}

[1] P. K. Pandey, M.L., Soupir, "Escherichia coli inactivation kinetics in anaerobic digestion of dairy manure under moderate, mesophilic and thermophilic temperatures." AMB Express, Vol 1, pp.1-10, 2011.

[2] S. Biswas, P. K. Pandey, T. B. Farver, "Assessing the impacts of temperature and storage on Escherichia coli, Salmonella, and L. monocytogenes decay in dairy manure." Biopress and Biosystem Engineering, vol. 39, pp. 901-913, 2016.
[3] California Deparment of Food and Agriculture (CDFA), "Dairy Digester Research and Development." https://www.cdfa.ca.gov/oefi/ddrdp/ (accessed on 9/10/2017).

[4] Ministry of New and Rnewable Energy (MNRE), "Biogas Program" http://mnre.gov.in/file-manager/UserFiles/faq biogas.htm (accessed on 9/10/2017).

[5] The EcoTyippiong Points Projects "China-Bioags." http://www.ecotippingpoints.org/our-stories/indepth/chinabiogas.html (accessed on 9/8/2017).

[6] Food and Agriculture Organization (FAO), "Biogas Institute of Ministry of Agriculture (BIOMA)." http://www.fao.org/south-southgateway/database/detail/en/c/288482/ (accessed on 9/1/2017).

[7] American Public Health Association (APHA), "Standard methods for the examination of water and wastewater," A.W.W.A., Water Environment Federation, 1999.

[8] R. H. Zhang, Tao J, Dugba, PN. "Evaluation of Two-stage anaerobic sequencing batch reactor systems for animal wastewater treatment, " Trans of ASABE, vol 43, pp 1795-1801, 2000.

[9] Wen-Chien Kuo, Wen-Lung Lai, "Treatment of kitchen waste using a mobile thermophilic anaerobic digestion system", Renewable Energy, vol 35, pp 2335-2339, 2010.

[10] Riau, V., M. Á. De la Rubia, and M. Pérez. "Temperature-phased anaerobic digestion (TPAD) to obtain class A biosolids: A semi-continuous study," Bioresource Technology, vol 101, pp 2706-2712, 2009. 